\title{
PIV measurements of chevrons on F400-series tactical aircraft nozzle model
}

\author{
James Bridges", Mark P. Wernet ${ }^{\dagger} \&$ Franco C. Frate \\ NASA Glenn Research Center, Cleveland, $\mathrm{OH}$
}

\begin{abstract}
Reducing noise of tactical jet aircraft has taken on fresh urgency as core engine technologies allow higher specific-thrust engines and as society become more concerned for the health of its military workforce. Noise reduction on this application has lagged the commercial field as incentives for quieting military aircraft have not been as strong as in their civilian counterparts. And noise reduction strategies employed on civilian engines may not be directly applicable due to the differences in exhaust system architecture and mission. For instance, the noise reduction technology of chevrons, examined in this study, will need to be modified to take into account the special features of tactical aircraft nozzles. In practice, these nozzles have divergent slats that are tied to throttle position, and at take off the jet flow is highly overexpanded as the nozzle is optimized for cruise altitude rather than sea level. In simple oil flow visualization experiments conducted at the onset of the current test program flow barely stays attached at end of nozzle at takeoff conditions. This adds a new twist to the design of chevrons. Upon reaching the nozzle exit the flow shrinks inward radially, meaning that for a chevron to penetrate the flow it must extend much farther away from the baseline nozzle streamline. Another wrinkle is that with a variable divergence angle on the nozzle, the effective penetration will differ with throttle position and altitude. The final note of realism introduced in these experiments was to simulate the manner in which bypass flow is bled into the nozzle wall in real engines to cool the nozzle, which might cause very fat boundary layer at exit. These factors, along with several other issues specific to the application of chevrons to convergent-divergent nozzles have been explored with particle image velocimetry measurements and are presented in this paper.
\end{abstract}

\section{Introduction}

The Navy has recently been studying the application of chevrons to overexpanded plumes from convergentdivergent (C-D) nozzles as a method of reducing the noise exposure of carrier crew and neighborhoods around Navy bases. NASA has collaborated with the Navy and its industry partner GE to investigate how previous experience with chevrons can be used to reduce jet noise in the overexpanded nozzle flow typical of tactical aircraft at takeoff. Specifically, the Supersonics Project within NASA's Fundamental Aeronautics Program has addressed the question of whether current physics-based aeroacoustic tools apply, or whether new predictive tools need to be developed.

Two things make applying chevrons to tactical aircraft different than typical commercial subsonic exhaust systems: tactical aircraft have actuated C-D nozzles with area ratios tied to throttle, and their throat to exit area ratio at takeoff is designed to be appropriate for cruise pressure ratio at altitude. Thus the nozzle flow is strongly overexpanded at takeoff. Flow leaves the nozzle surface at or near exit and curves inward, as shown in Figure 1. Also shown schematically in white, chevrons may have to penetrate quite far from the divergence flap plane, in red, to create vorticity. The other aspect that makes application of chevrons different is that the chevron geometric

\footnotetext{
${ }^{*}$ Acoustics Branch, NASA Glenn Research Center, Associate Fellow AIAA.

Optical Instrumentation Branch, NASA Glenn Research Center, Associate Fellow AIAA.

* Mechanical Engineer III, ASRC Aerospace, Inlet and Nozzle Branch, NASA Glenn Research Center, MS500ASRC 21000 Brookpark Road, Cleveland, OH 44135, AIAA member.
} 
parameters, such as penetration, vary relative to the flow as the area ratio of the convergent-divergent nozzle changes with throttle setting. Any fixed chevron design will have to work over a broad range of flow immersions and conditions, something that fixed commercial nozzles do not face.

One other complicating factor comes into play - the effect of the film cooling on the flow that interacts with the chevrons. This low momentum air bled into the nozzle through many small passages along the nozzle wall produces a significantly larger boundary layer than is usually encountered in designing chevrons for a convergent nozzle just because it is injected at comparable pressures to the main flow but at greatly reduced temperatures. In previous work by Seiner et al [1], the flow in the engine nozzle was simulated using RANS CFD and the boundary layer found to be a significant fraction of the flow profile. This insight raised questions regarding how well model-scale tests of chevrons, performed with a single-stream flow, would compare with the real engine. It even raised questions whether tactical aircraft jet noise could be properly modeled using small-scale experiments. Indeed, acoustic tests directly comparing the impact of chevrons on the tactical nozzle models described herein showed that there was a significant error in ignoring the bypass flow $\left[^{2}\right]$. The impact of this altered flow profile on the performance of the chevrons, and how to mimic its effect in typical single-stream jet rigs was a major objective of this test effort.

To obtain an understanding of how overexpansion of the flow in the C-D nozzle impact design of chevrons, first a series of Reynolds-averaged Navier-Stokes (RANS) computational fluid dynamics (CFD) runs were performed to see the flow lines from the baseline C-D nozzle, paying special attention to the separation at the nozzle exit. Second, a series of chevron designs were conceived which incorporated the usual parameters that have been found to be important in subsonic applications of chevrons, include penetration, length, width, tangency to the baseline nozzle surface, and azimuthal curvature. These CFD efforts are documented in a companion paper in this Aerospace Sciences Meeting [3]. From these runs it was determined that the first three were strongly significant in affecting the turbulence and mixing, and a series of chevrons were designed for a range of these three parameters in all combinations. Some experimentation led to the selection of the final parameter ranges and model-scale chevron inserts were fabricated to attach to an existing nozzle model. These chevrons were tested acoustically using a Modern Design of Experiments regimen that allowed the rigorous modeling of the noise impact of chevrons in the parametric space. These experiments are described in reference [4]. After studying the impact of the chevrons on far-field noise of the jet, a subset of the chevrons was tested using particle image velocimetry (PIV) to measure mean and turbulent velocities. These measurements, described herein, complement the acoustic measurements, help explain the acoustic impacts observed, and can guide further development of chevrons for tactical aircraft applications.
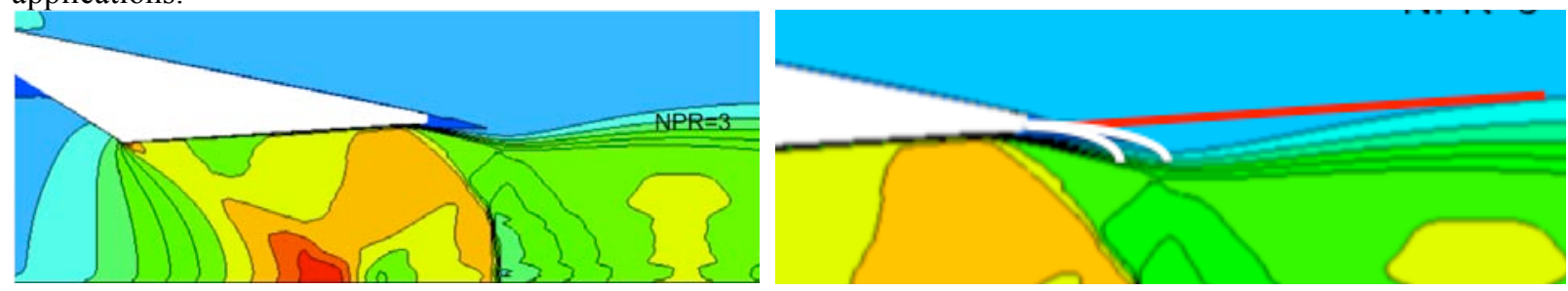

Figure 1 Mach number contours from CFD of F-400 series nozzle at Md $=1.65$ operating at $M=1.3$. In close-up view on right, red line shows datum line extending divergent flap angle, white curves are potential chevron profiles, showing how different lengths might interact with the shear layer.

\section{Test facility, model hardware, and instrumentation}

\section{A. Facility details}

The test was done at Nozzle Acoustic Test Rig (NATR) in the AeroAcoustic Propulsion Lab (AAPL) at NASA Glenn Research Center. The AAPL is a large, anechoic geodesic dome housing the NATR, a 53" diameter free jet tunnel that provides forward flight flow over the nozzle models. The nozzles were supplied with air streams at realistic pressures and temperatures using the dual-stream High Flow Jet Exit Rig. Somewhat unique to this test was the ability to model the impact of the cooling flow on the chevron performance. Figure 2 shows the bypass bleed holes in an F100 engine that are typical of tactical aircraft with afterburner exhausts, and schematically, the simple simulation of the bypass stream provided by the axisymmetric splitter of the model test rig. With a bypass-to-core area ratio of 0.2 the resulting mass bypass ratio of 0.3 was very close to the published values of the F404 and F414 engines. 

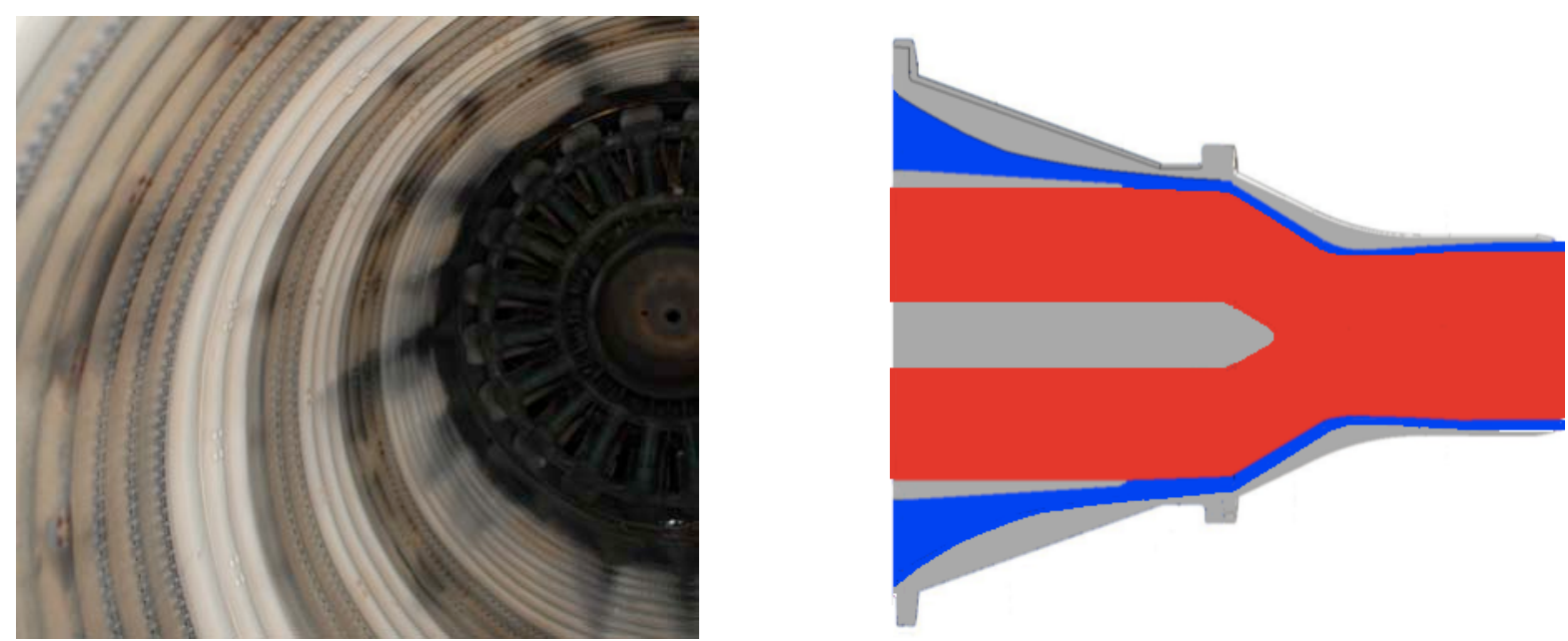

Figure 2 Simulation of bypass stream in nozzle. Left: bleed slots in tactical nozzle used to introduce bypass flow into nozzle. Right core-bypass split from internal axisymmetric splitter with area ratio 0.2 simulating the cooling film flow.

\section{B. Model hardware}

\section{GE F400-series model}

A model-scale nozzle developed by General Electric Aircraft Engines was used to mimic their F404 nozzle. This nozzle model consisted of two parts, a contraction section and a choice of divergent sections with design Mach numbers 1.5 (nozzle G5), and 1.65 (nozzle G6). The nozzle has 12 flaps/seals and an effective throat diameter of 4.454 inches. The equivalent exit diameters of the two divergent sections were 4.84 inches and 5.067 inches corresponding to design Mach numbers 1.5 and 1.65. Although it is hard to see in the photo (Figure 3) each flap has pockets on the inside tip where chevrons are bolted on.
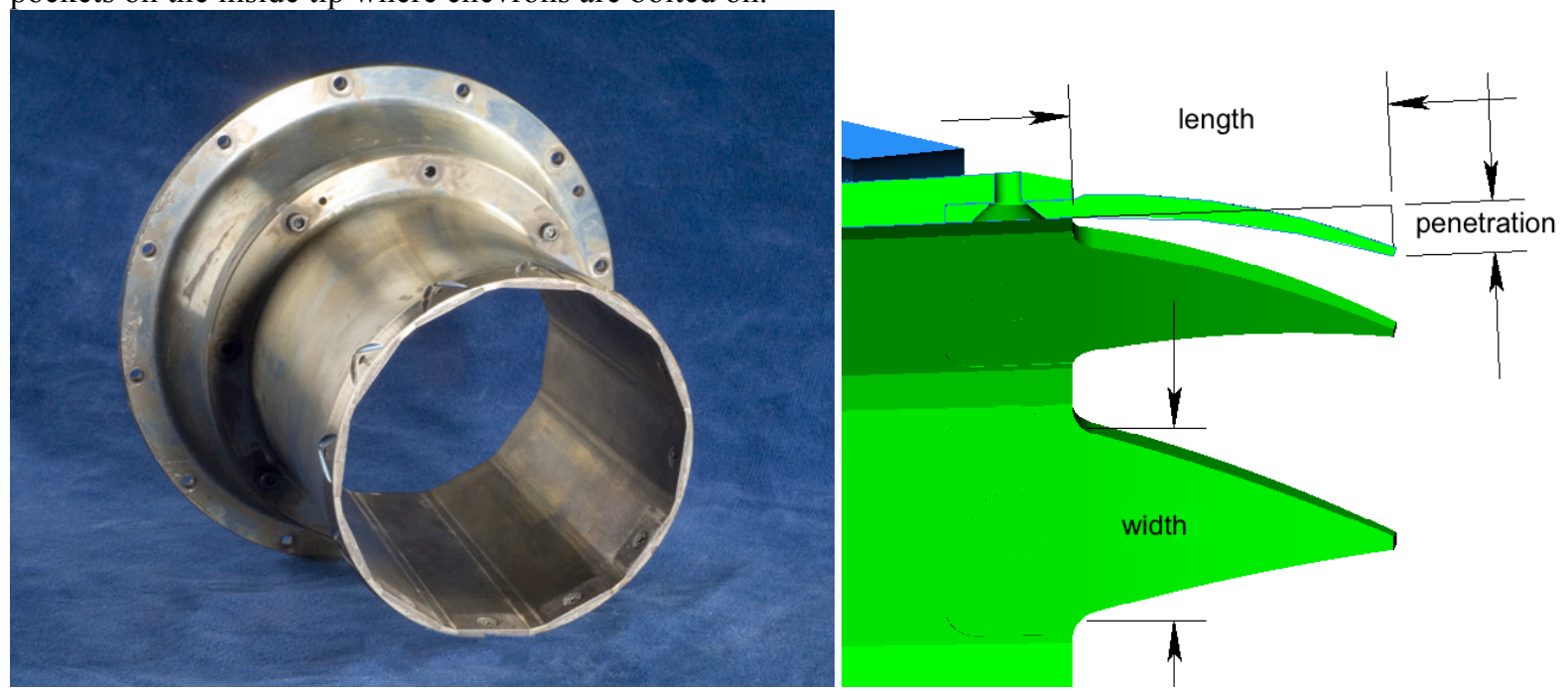

Figure 3 Model nozzle hardware. Left: photo of F-400 series model nozzle with blank fillers. Right: sketch defining chevron geometrical parameters.

\section{NASA Chevron designs}

The NASA MDOE parametric matrix used the parameters of Penetration, Length, Width, with the constraints that the chevrons be flat azimuthally, of triangular planform, and have a constant radius whose arc is tangent to the flap surface. The length is defined as the distance from the exit of the baseline nozzle to the tip of the chevron. The penetration is defined as the deflection of the tip from the surface extending from the upstream flap. The width was a percentage of the flap width, varying from $60 \%$ to $100 \%$ of the flap width. Penetrations varied from 0.3 to 0.6 inches relative to the flap line, and lengths from 0.75 to 1.75 inches from the baseline exit plane. Nomenclature for the

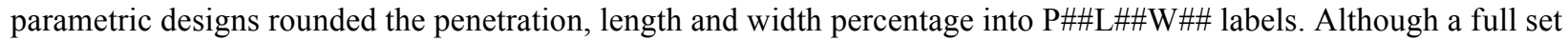
of parametric combinations was tested for noise, including the MDOE parametric centerpoint, the number of 
configurations tested with PIV was reduced to fit the test budget and schedule based on the acoustic results. PIV testing also included one variation in C-D area ratio along with excursions in flow conditions.

Figure 4 shows the chevron nozzle configurations tested relative to a parametric design space cube. The designs tested hit the least aggressive to most aggressive chevron designs along the diagonal. Because acoustic tests showed that penetration was the strongest parameter on jet noise we tested one direct variation of penetration. One additional design with smaller penetration was added as a low-risk design, having a penetration of 0.2 " and the same length and width as the centerpoint design. In effect four different penetration designs were tested, for which several comparisons will be made. Because the chevron parameter of penetration was measured relative to the divergent flap surface, variations in the angle of this surface change the effective penetration of the chevron. Therefore, while all the configurations shown in Figure 4 were tested on the G6 nozzle $\left(\mathrm{M}_{\mathrm{d}}=1.65\right)$, a limited number of tests were performed on the G5 nozzle $\left(\mathrm{M}_{\mathrm{d}}=1.5\right)$.

Table 1 Nozzle and chevron configurations used in test.

\begin{tabular}{|c|c|c|c|c|}
\hline Configuration & $M_{\text {design }}$ & Penetration & Length & Width \\
\hline G5 & 1.5 & & & \\
\hline G5 P02L13W08 & 1.5 & $0.20 "$ & $1.25 "$ & 0.8 \\
\hline G5 P05L13W08 & 1.5 & $0.45 "$ & $1.25 "$ & 0.8 \\
\hline G6 & 1.65 & & & \\
\hline G6 P02L13W08 & 1.65 & $0.20 "$ & $1.25 "$ & 0.8 \\
\hline G6 P03L08W06 & 1.65 & $0.30 "$ & $0.75 "$ & 0.6 \\
\hline G6 P03L18W10 & 1.65 & $0.30 "$ & $1.75^{\prime \prime}$ & 1.0 \\
\hline G6 P05L13W08 & 1.65 & $0.45 "$ & $1.25 "$ & 0.8 \\
\hline G6 P06L18W10 & 1.65 & $0.60 "$ & $1.75^{\prime \prime}$ & 1.0 \\
\hline
\end{tabular}

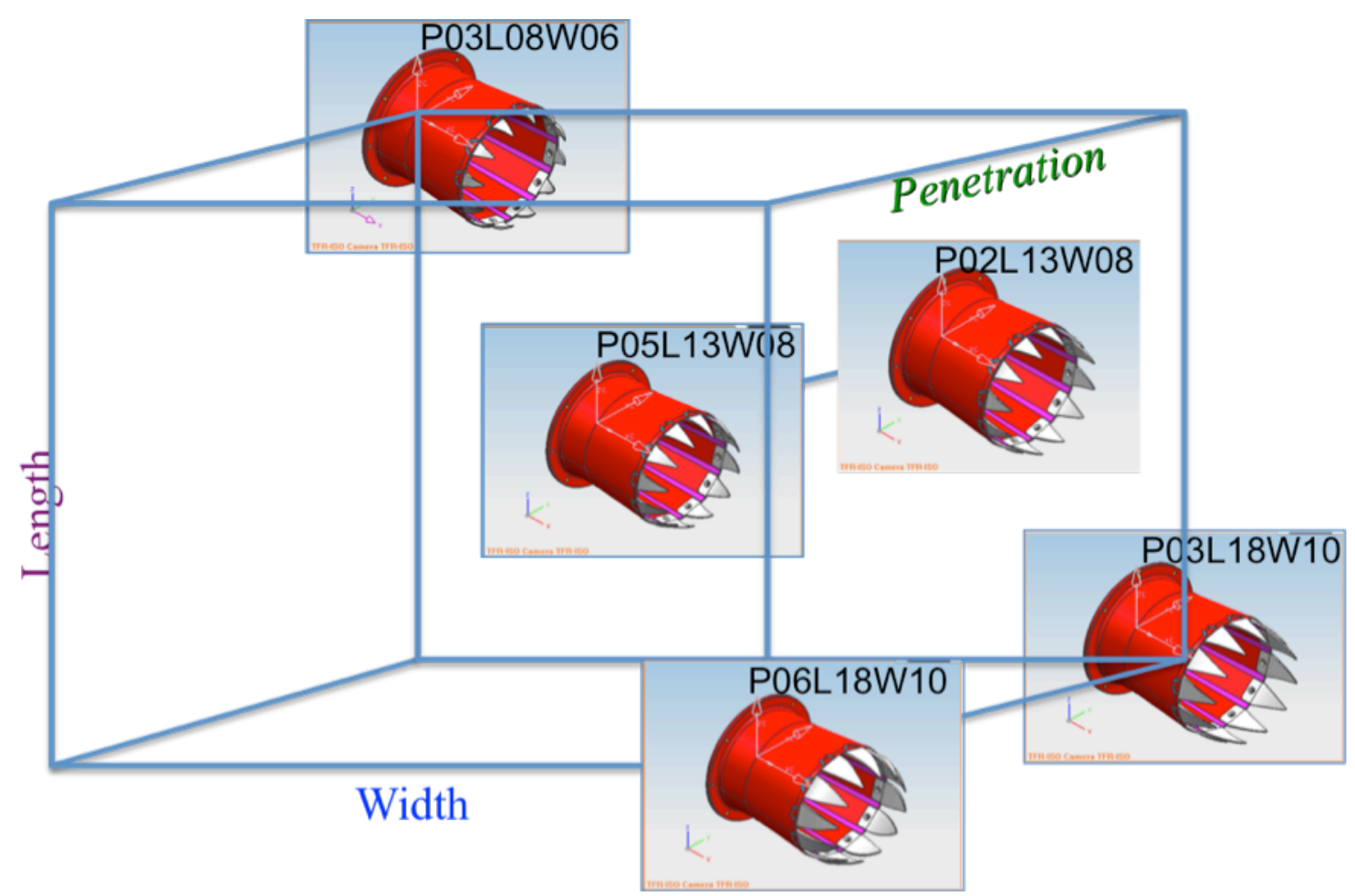

Figure 4 Chevron designs placed within the three-dimensional parameter space of Penetration, Length, and Width.

\section{PIV setup}

Data was acquired in two setups of the PIV system (see Figure 5): one mapping streamwise planes using two side-by-side cameras to obtain two-components of velocity including the centerline; and another mapping crossstream planes, with the cameras in a stereo configuration to obtain 3-components of velocity, concentrating near the nozzle to see the amount of axial vorticity produced by the chevrons. The axial locations measured were at $0.2,0.4$, 
$0.6,1.0,2.0$, and 5.0 exit diameters from the baseline no-chevron nozzle (excepting where planes were inside the length of the chevrons).

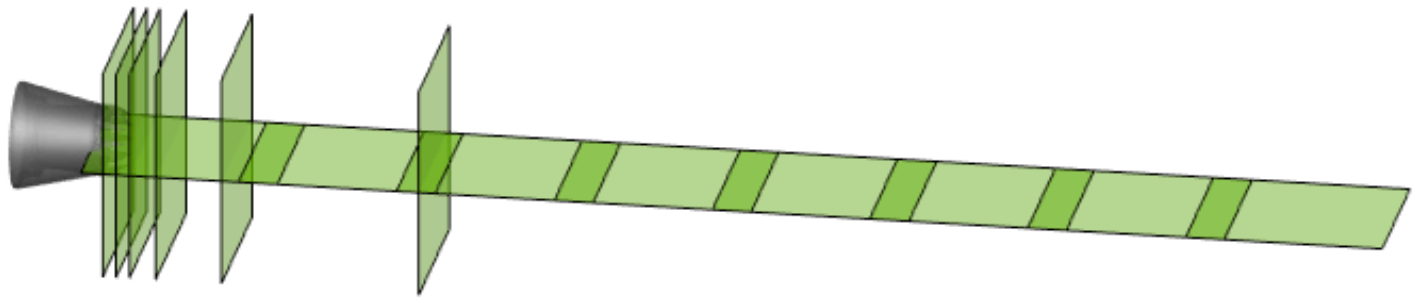

Figure 5 Planes relative to nozzle where PIV was acquired.

\section{3-D Stereo PIV Configuration}

The Stereo Particle Image Velocimetry (SPIV) system was configured to provide cross-stream measurements of the three-component velocity field from the test article. The entire SPIV system was mounted on a large traverse system to perform velocity plane surveys of the flow field. The SPIV system employed two high-resolution (4008x2672 pixel) cameras equipped with $180 \mathrm{~mm}$ focal length lenses and $8 \mathrm{~mm}$ extension tubes to provide a $300 \times 360 \mathrm{~mm}$ field of view. The cameras were mounted downstream of the model exit plane at nominally $\pm 45^{\circ}$ from the nozzle centerline. Stereo PIV calibrations were performed using a single plane target translated to 9 axial positions over a $\pm 2 \mathrm{~mm}$ range. A 4 th order polynomial was used in the image warping and a calibration verification operation was employed to insure the calibration overlapped the laser light sheet plane. The measurement plane was illuminated using a dual head $400 \mathrm{~mJ} / \mathrm{pulse} \mathrm{Nd}$ :YAG laser system. The laser beams were formed into $1 \mathrm{~mm}$ by 350 $\mathrm{mm}$ light sheets using cylindrical and spherical lenses. Both cameras were connected to a single computer system via a CameraLink PCI card and the 400 frame pair data sequences were acquired and streamed to disk at a rate of 2 frame pairs/camera/sec.

Since the AAPL is open to the environment during testing, the NATR could not be operated in complete darkness. To accommodate this situation, optical backdrops for the cameras were provided. These darkened camera backgrounds were positioned such that they were aligned with each camera view behind the measurement plane. The backgrounds were offset a suitable distance to minimize any influence on the ambient seeded flow distribution. The frame-straddle cameras used in the GRC PIV systems incorporate fast-acting shutters in front of the CCD arrays to minimize the duration of the frame-straddled second exposures to a nominal $12 \mathrm{msec}$ (down from the camera fixed $240 \mathrm{msec} 2^{\text {nd }}$ frame integration period). The complete stereo PIV system, including all cameras and backdrops, data acquisition computers, laser hardware and optics, were rigidly mounted on a large axial traverse located downstream of the nozzle exit plane, which maintained a fixed position during each nozzle test. The travel range of the traverse was approximately $2.5 \mathrm{~m}$, with a positioning accuracy of $1.0 \mathrm{~mm}$. Re-zeroing of the traverse to coincide with the trailing edge of individual chevron nozzles was aided by the installation of a calibration target, required in stereo PIV, on a fixture secured to the traverse. When operating at elevated temperature setpoints, with an accompanying axial growth of the entire test rig following cold start-up, traverse re-zeroing adjustment was required and performed immediately after reaching steady state flow conditions by optical means, using the current camera nozzle views to reference known chevron trailing edge tip locations at the nozzle exit planes.

\section{2-D Streamwise PIV Configuration}

To maximize the field of view while maintaining high spatial resolution PIV vector maps, a dual side-by-side camera configuration was used to acquire streamwise planes of data. The $4008 \times 2672$ pixel PIV cameras were used with the 4008-pixel axis oriented vertically. The cameras were equipped with $180 \mathrm{~mm}$ focal length lenses and positioned so that their fields of view overlapped by $25 \mathrm{~mm}$. Both cameras were connected to a single computer system via a CameraLink PCI card and the 400 frame pair data sequences were acquired and streamed to disk at a rate of 2 frame pairs/camera/sec. A flat, stereo PIV-style calibration target was used to calibrate and register the two cameras using a fiducial mark in the overlapping region of the cameras' field of view. The physical registration of the two cameras was used in the setup of the vector processing grids in the left and right camera images so that no interpolation was required in the merging of the left/right vector maps. The final merged camera vector map covered an area of $380 \times 325 \mathrm{~mm}$.

3. Vector Processing

Velocity vector maps for each camera were computed from the image pairs using the in-house PIVPROC software. The software utilizes conventional multi-pass PIV cross-correlation processing algorithms and incorporates error detection based on image correlation signal to noise ratio. First-pass interrogation region sizes of 64 x 64 pixels on 32 pixel centers and final-pass interrogation region sizes of $32 \times 32$ pixels on 16 pixel centers were 
used to process image pairs from the cameras in both stereo configurations. For the cross-flow measurement planes near the nozzle, Symmetric Phase Only Filtering (SPOF) was employed to reduce the effects of flare light on the nozzle models directly behind the measurement planes. Without the SPOF processing, images with nozzles illuminated by flare light behind the plane of interest generally produce regions in and around the potential core flow with invalid vector measurements. The SPOF processing technique was not utilized with the axial-flow measurement planes as the nozzle models do not appear in the field of view. All of the data were processed using LogLut intensity filters and Subregion Distortion processing. For the 3-D PIV data, the left/right vector maps were processed with an additional in-house code to generate the 3-D vector maps.

\section{Test matrix}

The test focused on the effect of expansion ratio and NPR on the effectiveness of the chevron designs. Figure 6 shows the flow condition matrix used in terms of aero Mach number vs acoustic Mach number (velocity). Given that most of the CFD-based design was done at $\mathrm{NPR}=3\left(\mathrm{M}_{\text {ideal }}=1.36\right)$ this was a key flow case. A high $\mathrm{M}_{\text {ideal }}$ case of $\mathrm{M}_{\text {ideal }}=1.48$, still overexpanded for the $\mathrm{G} 6$ nozzle $\mathrm{M}_{\mathrm{d}}=1.65$, was studied for all chevron configurations. Contrasting the flow in the G6 nozzle with the flow from the G5 nozzle $\left(\mathrm{M}_{\mathrm{d}}=1.5\right)$ showed the impact of overexpansion on the chevron impact. Additional flow conditions at $\mathrm{M}_{\text {ideal }}=1.5$ but at different core stream temperature ratios highlighted the role velocity plays in the chevron designs and were conducted for one chevron configuration (P06L18W10) only.

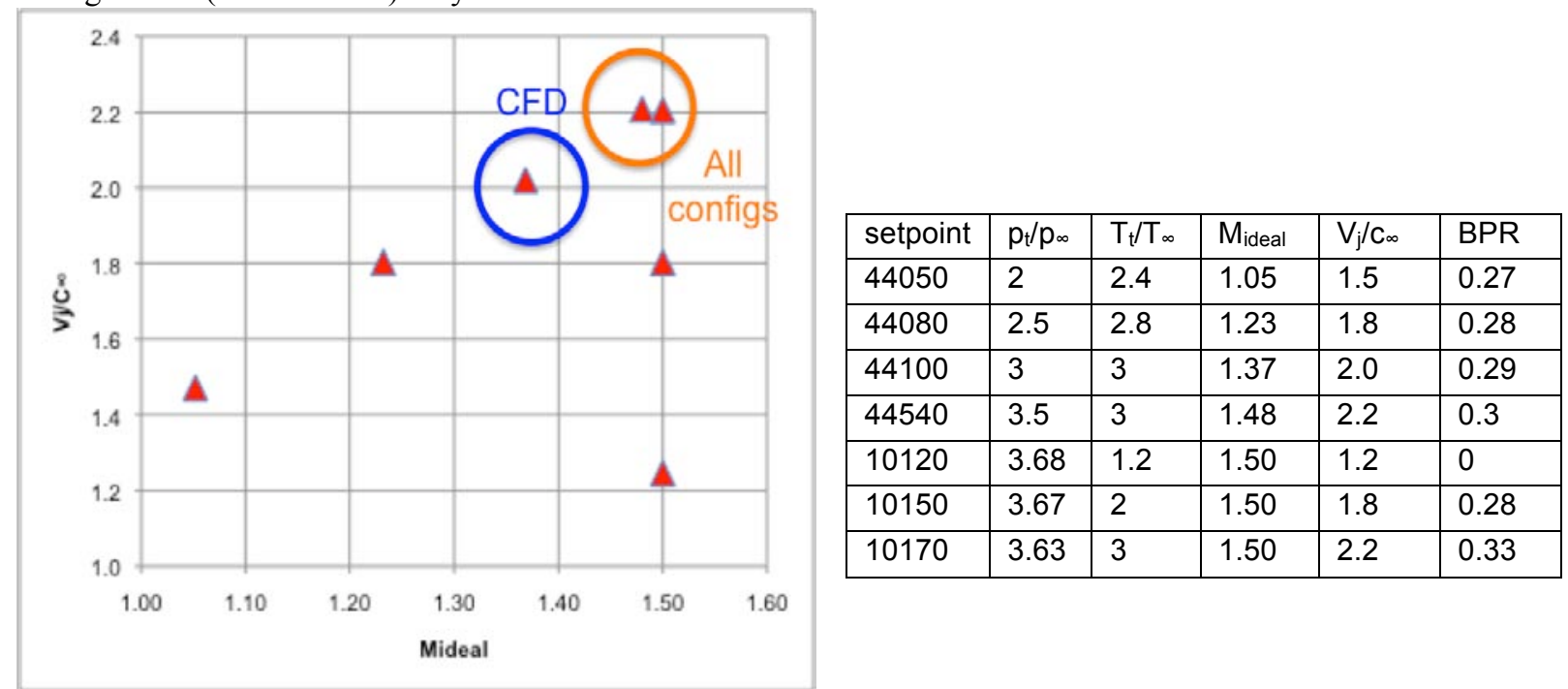

Figure 6 Flow conditions tested as given by ideally expanded Mach number $M_{\text {ideal }}$ and acoustic Mach number $V_{j} / c_{\infty}$.

\section{Results and Discussion}

In presenting results first the role of the low speed boundary layer will be addressed, followed by presentation of the impact of the various chevron parameters.

\section{A. Exit velocity profiles-dual flow vs single flow}

For most of the PIV measurements made the rig was run with bypass stream of matching pressure and effectively ambient temperature. Ideally, measurements of the flow profile with and without the cooling bypass stream would have been made and directly compared to determine the impact of the bypass stream relative to a single-stream hot jet. However, the jet rig could not be run for long without some cold air flowing in the bypass duct. Comparisons could only be made with a single-stream jet where the stream was cold, accomplished by running the rig with both streams cold. This single-stream flow, having no internal mixing layer, was then compared with the dual stream flow by normalizing the exit velocity profile of each flow by the ideally expanded velocity of the core stream.

The impact of the bypass flow on the exit velocity profile is addressed by the plots in Figure 7 of the baseline nozzle $\mathrm{G} 6$, operated overexpanded at $\mathrm{M}_{\text {ideal }}=1.5$. In the plot on the left, mean axial velocity profiles are plotted versus radius normalized by the exit equivalent diameter, $\mathrm{D}_{\text {exit }}$. The plot on the right shows the corresponding profile of axial turbulence intensity. Both profiles are normalized by the fully expanded core flow velocity. The flow at this 
location $\left(\mathrm{x} / \mathrm{D}_{\text {exit }}=0.07\right)$ is not ideally expanded; in fact, the profile crosses an oblique shock coming from within the nozzle and creating a nonuniform velocity across the nozzle exit in both flows. Note the fuller profile for the single stream $(B P R=0)$ mean flow near the lip line. The velocity deficit on the dual-stream flow $(B P R=0.3)$ profile is the legacy of the cold, low-speed bypass air from the small annulus upstream of the nozzle. The dual-stream aspect is especially evident in the turbulence profiles where the shear layer between the cold and hot streams is evident in the dual-stream case as the second peak of turbulence at $\mathrm{r} / \mathrm{D}_{\text {exit }}=0.4$. The single-stream flow has only a single, clear peak in turbulence, of roughly $15 \%$ of fully expanded velocity, consistent with most measurements of turbulence in laboratory jets.

The difference between the two flows at the nozzle exit is of interest in jet noise for two reasons. First, because the noise processes are dependent upon the turbulence and the second shear layer should provide an additional source of noise not present in the single-stream flow. Second, in the application of chevrons for noise reduction the amount of vorticity created by the chevron is dependent upon the velocity field into which the chevron protrudes. For example, in the current test program the tips of the various chevrons extend into the flow to roughly $\mathrm{r} / \mathrm{D}_{\text {exit }}=$ 0.4-0.45. Although the flow is not a simple axial flow due to the shocks, it seems likely that the chevrons would produce much greater impact on the $\mathrm{BPR}=0$ flow than on the $\mathrm{BPR}=0.3$ one because the velocity is so much greater. On the other hand, it is possible that, although the velocity of the cold flow is lower, the increased density of this low-speed fluid would make up for it because it has equivalent momentum.
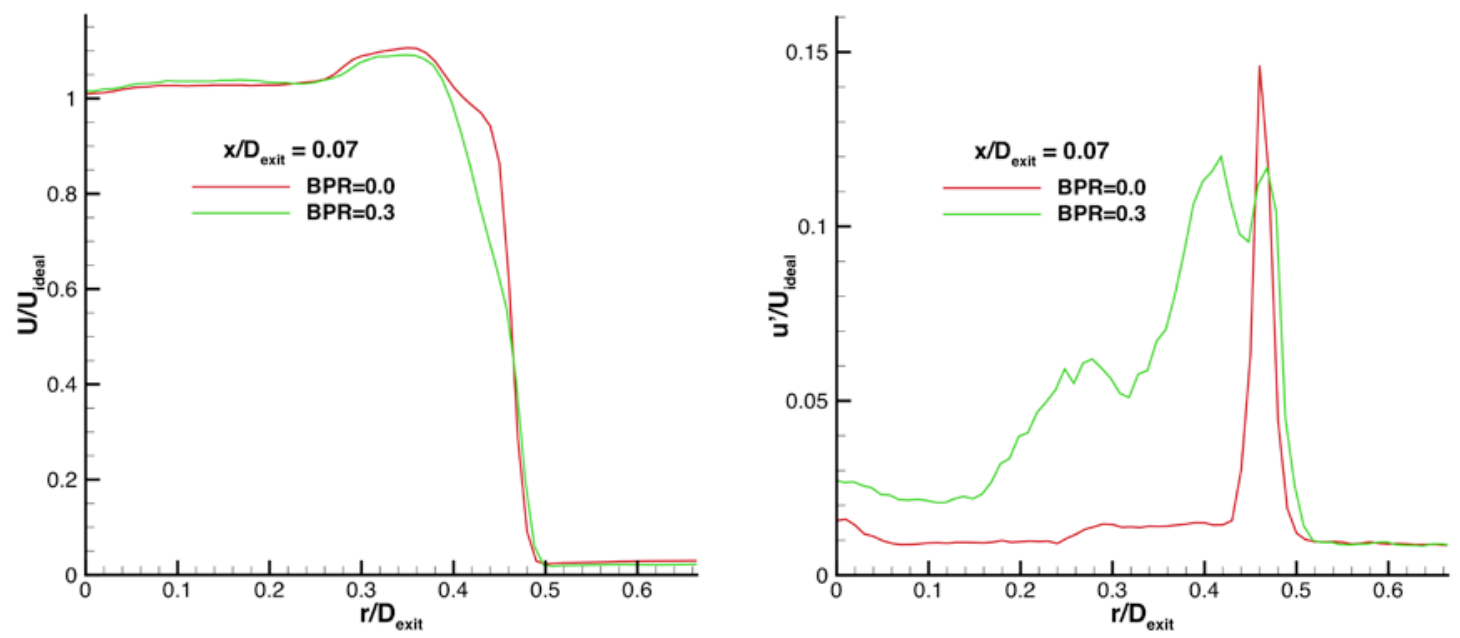

Figure 7 Radial profiles of mean (left) and rms (right) axial velocity of a $M_{\text {ideal }}=1.48$ jet, acquired at $x / D j=0.07$. Velocity profiles normalized by ideally expanded velocity. BPR $=0$ data acquired at single-stream (cold) condition and compared with data from $B P R=0.3$ dual stream $\left(T_{t} / T_{\infty}=3\right)$ to demonstrate velocity deficit caused by bypass stream.

\section{B. Impact of chevrons}

To assess the impact of the chevrons on the flow, the first presentation of results will be using the cross-stream planes of data. Figure 8a-f gives a quick overview of how the plume varied with increasingly aggressive (top to bottom) chevron designs. All chevron configurations are shown for the same flow condition, $\mathrm{M}_{\text {ideal }}=1.48, \mathrm{~V}_{\mathrm{j}} / \mathrm{c}_{\infty}=$ 2.21. Each plot of the figure shows mean axial velocity and axial vorticity in the different cross-sections for one chevron configuration (including the base, no-chevron configuration, Figure 8a). In each cross-section, the mean axial velocity is represented by contour lines and by the shape of the cross-sections as data below U/Uid $=0.1$ have been blanked in the plots. Judging by the radial extent of this lowest contour level, the greatly enhanced spreading of the chevrons with increased penetration is apparent. In all cases there are 12 lobes to the contour, although this is very subtle for the first few chevron configurations. It is dangerous to make too much of the velocity levels as the variations in velocity may result from changes in the shock structure as much as the enhanced mixing of the chevrons.

The axial vorticity is represented in the color contours of each cross-section with values given by the color bar. The color bar was intentionally kept the same between plots, which results in the vorticity values being clipped in the most extreme chevron configurations. This presentation does allow direct comparison of the vorticity between configurations, including the vorticity present in the base, no-chevron configuration (Figure 8a). Here, the facets 
representing the flaps of the variable area nozzle produce a measurable amount of axial vorticity, comparable to that of the lowest penetration chevron directly beside it (P02L13W08, Figure 8b).
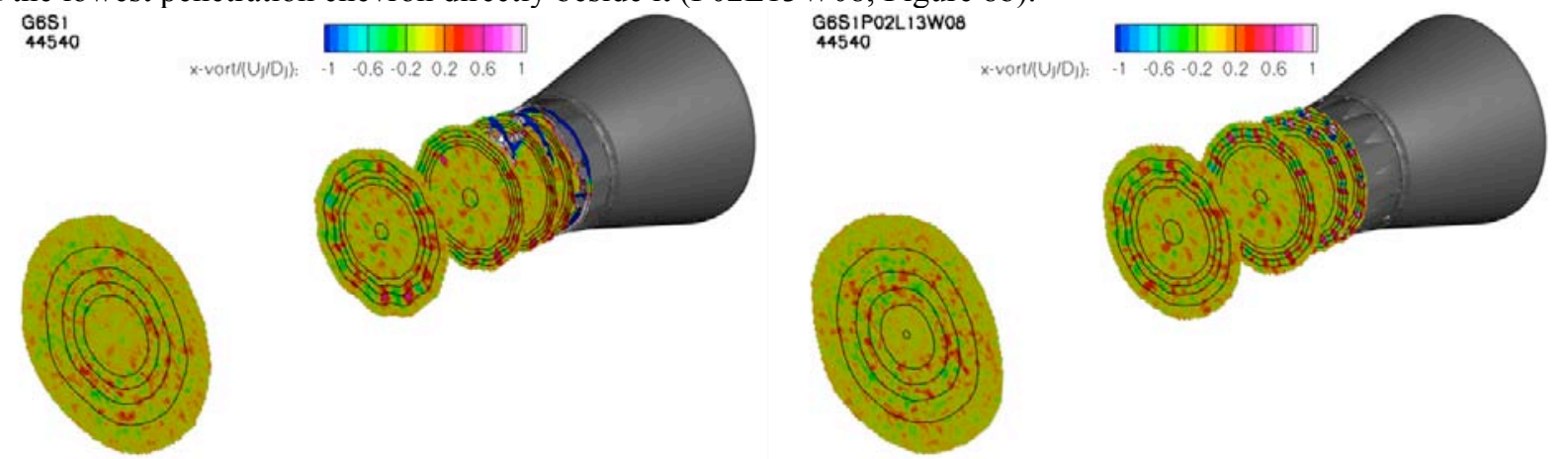

(a)

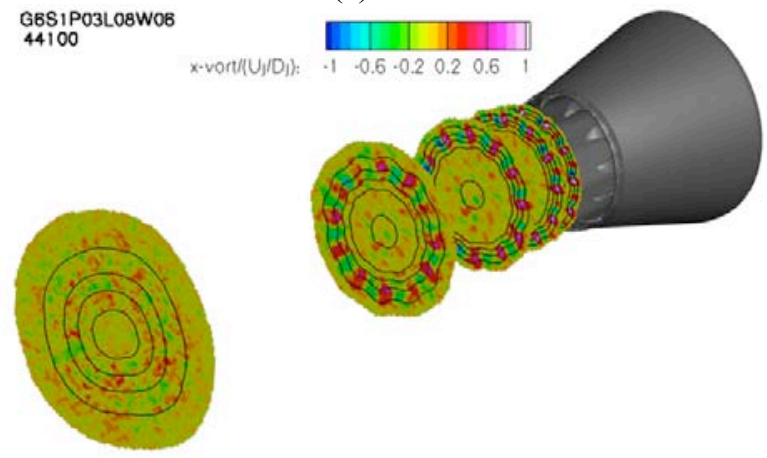

(c)

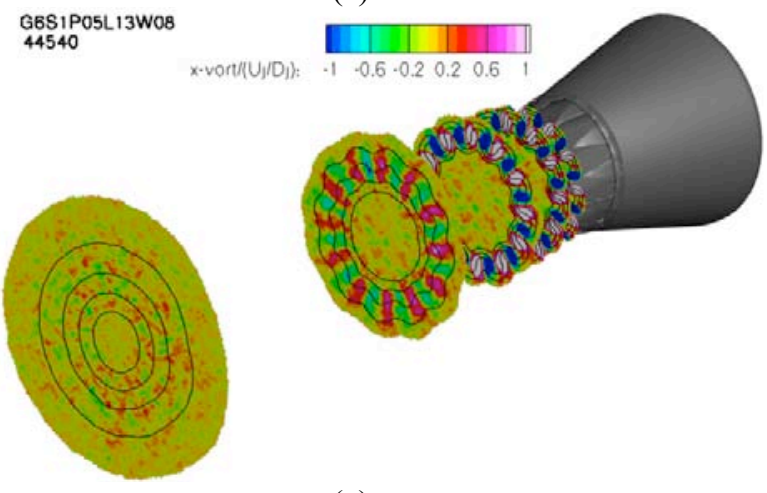

(e)

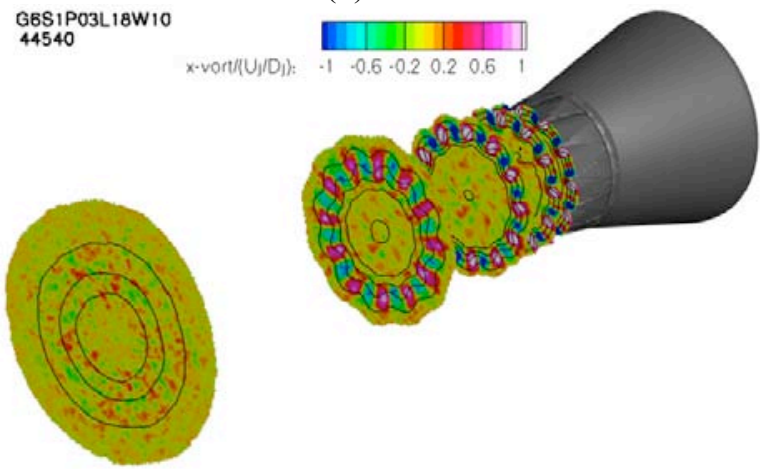

(d)

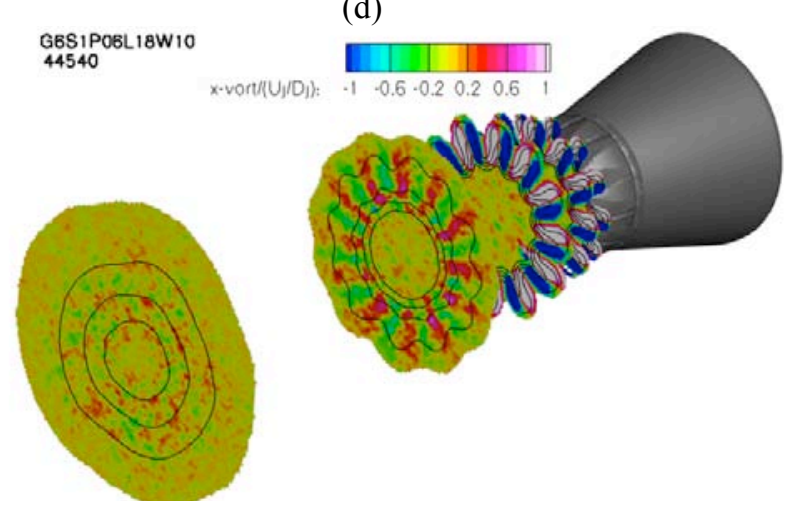

(f)

Figure 8 Cross-stream contours of mean streamwise velocity (black lines) and streamwise vorticity (color fills) for all configurations tested: (a) Baseline nozzle, (b) P02L13W08, (c) P03L08W06, (d) P03L18W10, (e) P05L13W08, (f) P06L18W10. NPR =3.2, $T_{t, c} / T_{\infty}=3.2$. Color fills cut off at $U / U_{j}=0.1$.

Focusing on the $\mathrm{x} / \mathrm{D}_{\text {exit }}=2$ plane (next to last plane going downstream) the peak vorticity is around \pm 0.4 for the base nozzle, increasing to 0.6 by the P03L08W06 (Figure 8c) configuration with more penetration. Increasing the length and width of the chevron (P03L18W10, Figure 8d), the vorticity at $\mathrm{x} / \mathrm{D}_{\text {exit }}=2$ has peak values of \pm 1 , the highest of all the configurations. The configuration with higher penetration and same length and width (P06L18W10, Figure 8f) shows larger vortices and greater spread, but the peak at $\mathrm{x} / \mathrm{D}_{\text {exit }}=2$ is diminished, perhaps because the vorticity is so extended in the radial direction. Visually, the vorticity plots of configurations P03L18W10 (Figure 8d) and P05L13W08 (Figure 8e) are rather similar. They seem to trade penetration of chevron against length and width to achieve similar vorticity. There is a significantly reduced peak vorticity at $x / D_{\text {exit }}=2$ in the configuration with higher penetration, and the vorticity at $\mathrm{x} / \mathrm{D}_{\text {exit }}=1$ is slightly more elliptic in its contours as well. This implies that increasing penetration may produce more direct spread, but that the vorticity is not as stable and breaks down closer to the nozzle, limiting the amount of mixing done by the vorticity downstream. 
Another notable feature of these plots is that there is no discernable organized axial vorticity at $\mathrm{x} / \mathrm{D}_{\text {exit }}=5$ in any of the configurations. Presumably the vorticity has diffused by this point downstream in all configurations.

4. Impact on mean velocity on centerline

Not readily observable in the cross-sections is the impact of the chevrons on the shock structure of the jet plume. Figure 9 shows plots of the mean axial velocity in the streamwise plane of measurement, illuminating the shock structure of the first few nozzle diameters. A significant feature of the shock structure of the baseline nozzle is the presence of a second shock train originating at the sharp throat, producing the multiple-peaked shock pattern on the centerline[5]. This second shock train is not discernable from external measurements but is clear from CFD done in designing the experiment. The dual-shock system of the external shock pattern confirms this feature in the baseline nozzle (Figure 9a).As expected from the previous analysis, the P02L13W08 configuration (Figure 9b) produces little change in the flow. The shorter version of the P03 penetration chevron (Figure 9c) seems to have reduced the strength of the external shock system, while the longer version (Figure 9d) seems to have weakened the internal shock system. Both are weakened by the P05L13W08 chevron (Figure 9e), while the internal shock is completely obliterated by the most intrusive chevron P06L18W10 (Figure 9e).

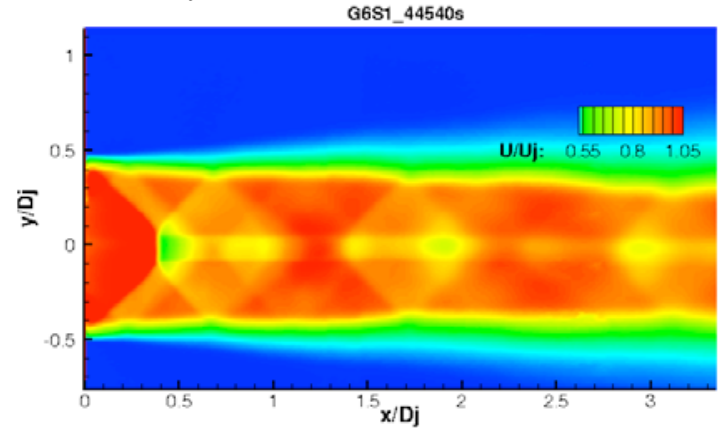

(a)

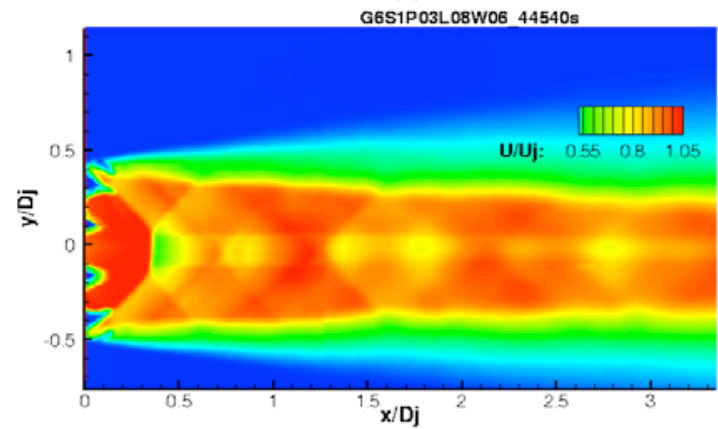

(c)

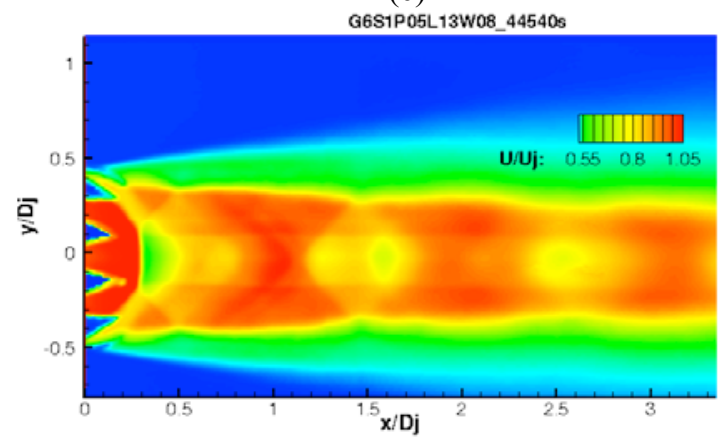

(e)

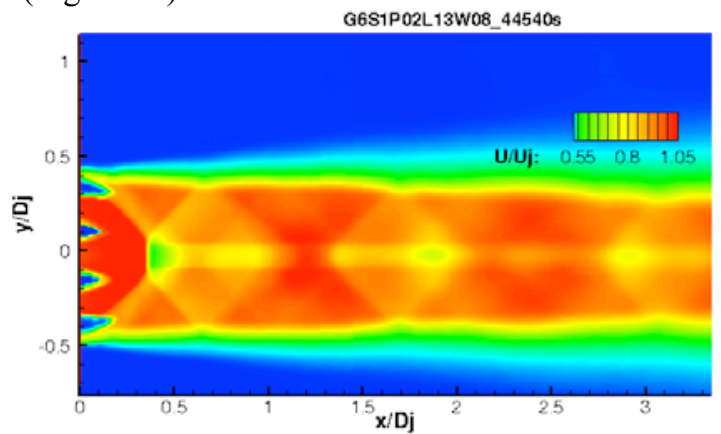

(b)

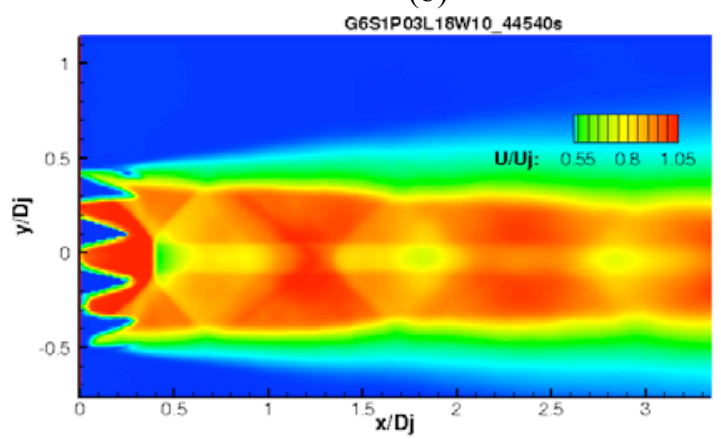

(d)

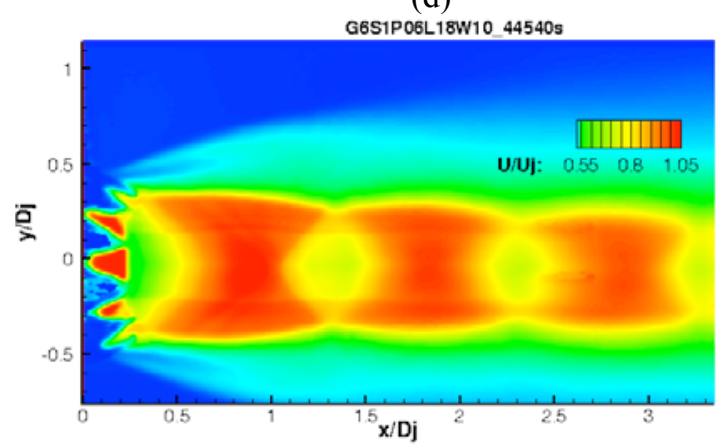

(f)

Figure 9 Contours of mean streamwise velocity for all configurations tested: (a) Baseline nozzle, (b) P02L13W08, (c) P03L08W06, (d) P03L18W10, (e) P05L13W08, (f) P06L18W10. NPR =3.2, $T_{t, c} / T_{\infty}=3.2$.

At this point the cause of the abrupt change in shock structure with increasing nozzle blockage is not clear. The shock structure of the three most intrusive chevrons does not show this second series of shocks very strongly, so perhaps the blockage is enough to eradicate the internal shock, leaving a more simple shock pattern. In CFD studies of different chevron designs it was noted that if the chevron penetration was large enough the effective area could be 
reduced enough to unchoke the throat of the nozzle. When one considers (see Figure 8) the convoluted shear layer produced by these most intrusive chevrons, however, simple shock patterns is not what is expected. What is clear from Figure 9 is how important it is not to interpret the velocity cross-sections of Figure 8, taken at fixed axial positions, as a measure of enhanced mixing.

By overlaying plots of centerline mean velocity we see the impact of chevrons on potential core length and on the shock structure. See Figure 10, where centerline velocity for most of the configurations has been plotted for the first 20 jet exit diameters to see changes in potential core length.

To orient, look for the black line that is the velocity profile of the baseline configuration. It has the longest potential core. The least penetration chevron (P02L13W08) makes little impact on this. With the next increase in penetration we see a significant decrease in potential core. From this (and Figure 8) it appears that the P02L13W08 chevron is not penetrating and/or blocking the flow enough to cause any change in the jet mixing, while P03L08W06 and all the other configurations are. The figure also shows how the shock structure of the baseline and P02L13W08 and P03L08W06 chevrons exhibits the two-train systems, while the other configurations do not.


Figure 10 Profiles of mean streamwise velocity on centerline of jets with different chevrons. Large range of $x / D j$ on left plot shows change in jet centerline decay, close up on right plot allows inspection of changes in shock structure.

\section{Impact on turbulence distribution}

From the point of jet mixing noise, the turbulence is key. In Figure 11 the axial component of turbulence, $u^{\prime} / \mathrm{U}_{\mathrm{j}}$, is plotted for streamwise cuts along the jet centerline. A side view of the nozzles with the various chevron configurations is shown alongside the plot of its turbulent field for a visual reference. A few notes are in order: First, note that the shocks are visible as slightly elevated 'turbulence' in the potential core of the jets. There was not a degradation in data quality in these locations and the low levels of variation in velocity from the mean was apparently caused by unsteadiness in the shock location. Second, the missing patches in the baseline configuration were regions near the edge of the PIV fields of view and data quality was locally low; hence these data were blanked and there is no reason to believe that the actual turbulence exhibited any modulation affiliated with this pattern. Similarly, the 'arcs' visible around $\mathrm{x} / \mathrm{D}=15$ in the last plot of the figure are spurious and should be ignored. Third, the 'bulge' in the shear layer at 1D in the last plot was largely because the plane of measurement was slightly off centerline and was cutting through the fold of the lobed shear.

For reference, peak values of this quantity for a single-stream subsonic jet is usually found to be around $14-15 \%$, so the peak values observed here were in line with values found in those jets. Scanning through the chevron configurations from least to most aggressive (top to bottom in Figure 11) there is a shift in distribution of turbulence from downstream to near the nozzle. This is also observed in application of chevrons to subsonic jets. Likewise, the peak turbulence intensity near the end of the potential cores is somewhat reduced with application of chevrons, matching expectations from subsonic jets. 

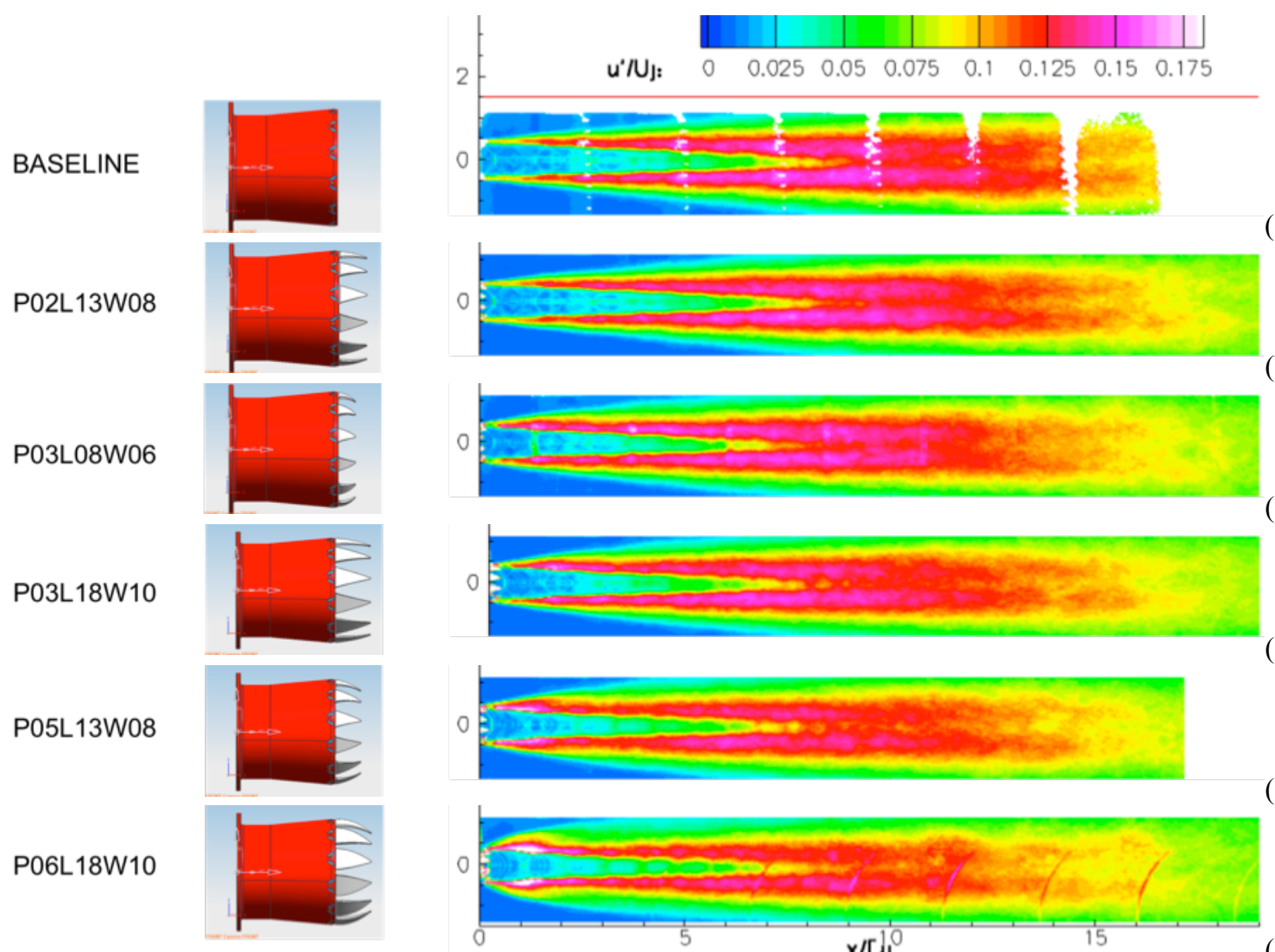

(a)

(b)

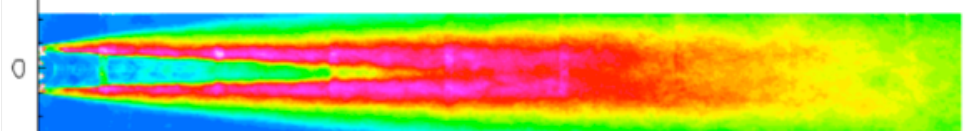

(c)

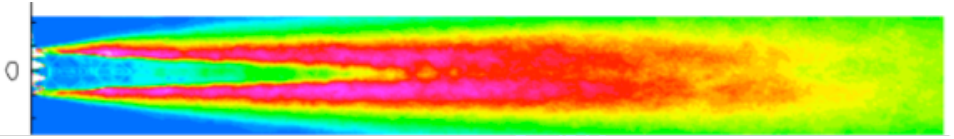

(d)

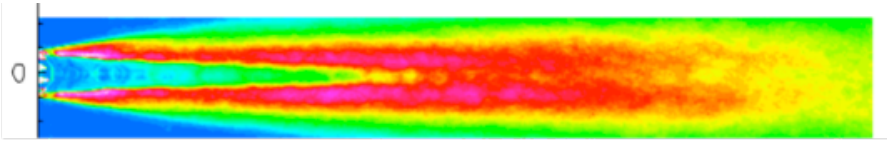

(e)

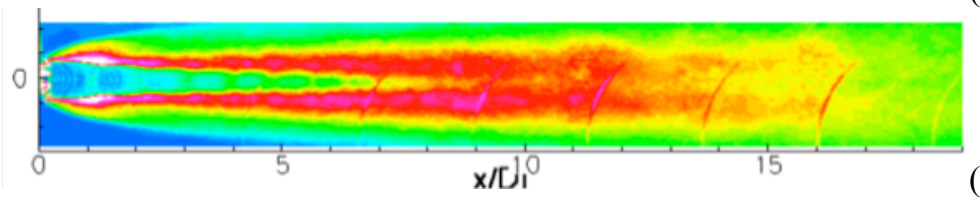

(f)

Figure 11 Streamwise cuts of streamwise turbulence intensity u'/Uj for different chevrons.

Perhaps, more directly applicable to jet mixing noise is the integration of turbulent kinetic energy (TKE) over axial slices. In many acoustic analogy formulations the noise from a particular region is proportional to $\mathrm{TKE}^{7 / 2}$ [6], so the integral of TKE over axial planes could be considered analogous to noise source strength $\mathrm{Q}$ (in $\mathrm{dB}$ ) as a function of axial location:

$$
Q(x) \sim \log \int_{A(x)} T K E / U_{j}^{2} d A .
$$

The plot of integrated TKE at each axial cross-section is shown in Figure 12. From this plot the impact of chevrons is to reduce noise sources aft of the potential core while strengthening noise near the nozzle. This is trend one finds in subsonic application of chevrons as well. The impact on broadband shock noise through modification of the shocks and the turbulence is not as simple and no attempt to generalize the acoustic impact of the chevrons on this source based on the flow features measured here will be attempted.

Note that after doing the integral across the plume the P02 chevrons do show some, small, benefit at aft angles with no discernable difference near the nozzle. A more detailed error analysis needs to be performed to determine if this is a statistically significant result. 


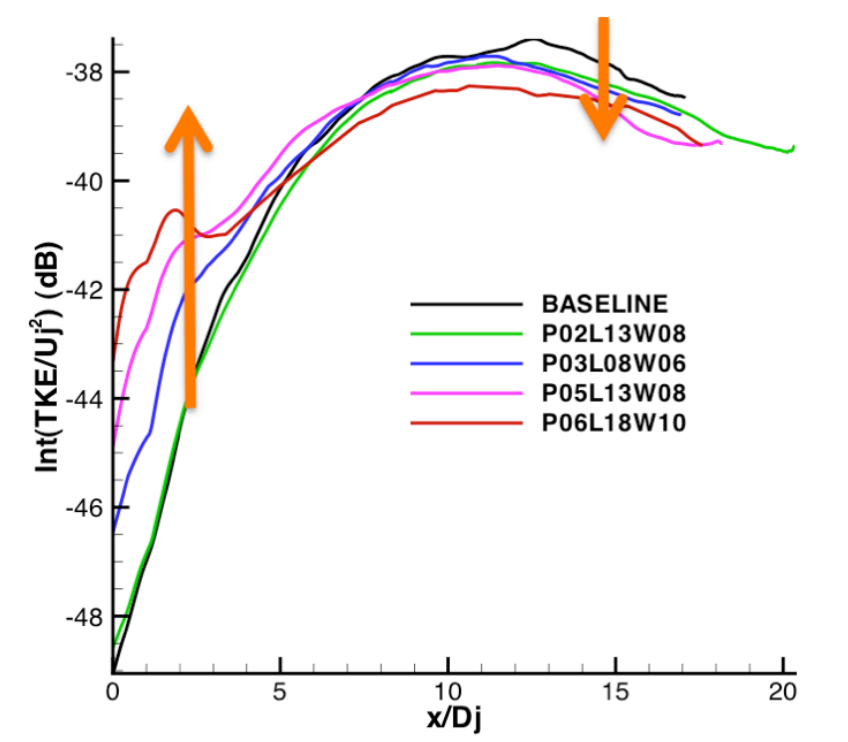

Figure 12 Plot of turbulent kinetic energy integrated over streamwise cross-section as a function of downstream distance for different chevrons. Units are $d B$ re 1.

\section{Variation of chevron impact with $\mathbf{M}_{\text {ideal }}$}

In conversations about the effect of chevrons on supersonic jets, there have been conflicting inferences form acoustic observations whether the chevron lengthened or extended the potential core in these flows. In the data presented above it appears that chevrons shorten the potential core for these overexpanded jets. However, the data presented above was only for the case where the $\mathrm{G} 6$ nozzle $\left(\mathrm{M}_{\mathrm{d}}=1.65\right)$ was run at $\mathrm{M}_{\text {ideal }}=1.5$ flow conditions. In the following data will be presented that shows that the change in potential length for a given chevron varies with the degree of overexpansion. In Figure 13 streamwise plots of mean axial velocity are shown for the G6 nozzle operated at different $\mathrm{M}_{\text {ideal }}$ conditions. To directly evaluate the impact of the chevron (in this case the P06L18W10 configuration) each plot has the plume of the baseline configuration on the bottom half with the plume of the chevron configuration on the top half. From these plots comparisons can be made on the how chevrons impact the shocks and potential core over a range of overexpanded Mach numbers.

The impact of chevrons on the length of the potential core is made apparent by the discrepancy between top and bottom halves along the centerline of each plot. The effect of the chevron flips with $\mathrm{M}_{\text {ideal }}$, from initially lengthening the potential core length at low $\mathrm{M}_{\text {ideal, }}$, to reducing it at high (but still overexpanded) Mach number. Data was acquired for only one chevron configuration at all $\mathrm{M}_{\text {ideal }}$, so this trend cannot be assured for less aggressive chevron designs. It has been shown above that near ideally expanded conditions the chevrons do reduce the potential core. If nothing else these measurements show the danger in over simplifying the impact of chevrons on nozzles with such complicated flow features as these overexpanded nozzles have. 


\section{$M_{\text {ideal }}=1.05$}

$M_{\text {ideal }}=1.23$

$M_{\text {ideal }}=1.37$

$M_{\text {ideal }}=1.48$
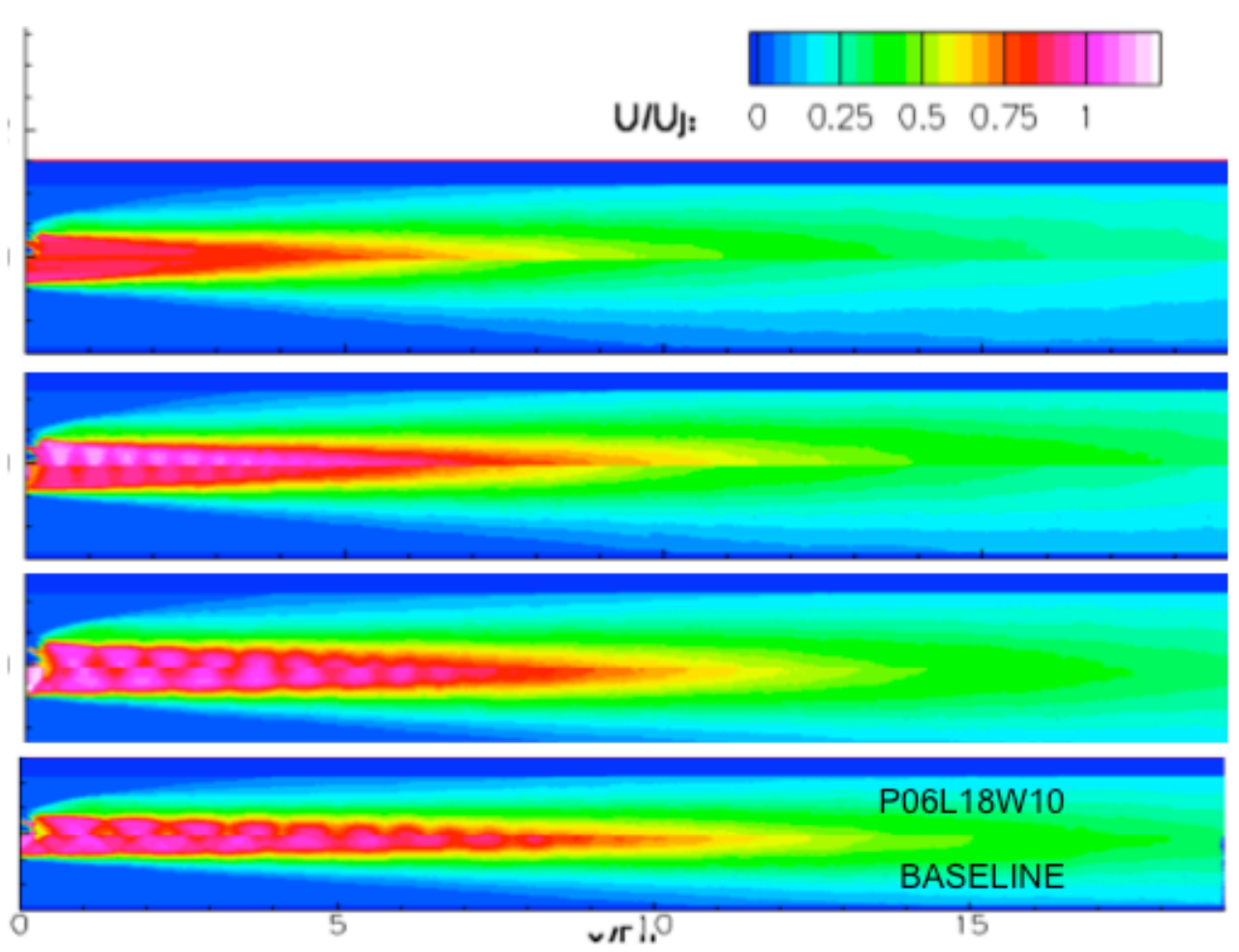

Figure 13 Plots of mean streamwise velocity, showing shock structure, for different flow Mach numbers. Each Mach number plot has the baseline nozzle flow on the bottom half and the P06L18W10 chevron flow on the upper half for direct comparison.

\section{Impact of nozzle divergence angle}

In operation, the nozzles of tactical aircraft change the area ratio of their convergent-divergent nozzles, and any chevron applied to the nozzle will operate in a wide range of geometric configurations. Consider what happens when the nozzle is actuated to a different A8/A9 ratio. The divergent flaps change angle, the $\mathrm{M}_{\mathrm{d}}$ changes, everything changes. In these tests this effect was studied by applying a low penetration chevron on nozzles with different divergence sections, mimicking a change in nozzle aspect ratio for a given chevron design.

Varying the divergence angle of the flaps on the nozzle has two effects: first the exit area changes making the flow less overexpanded, and second the chevrons rotate into the flow a bit more. Both cause the chevrons to have more impact on the flow. Figure 14 documents the streamwise cut of axial turbulence from chevron P02L13W08 on G5 and G6 nozzles, both run at $\mathrm{M}_{\text {ideal }}=1.48$. On the G6 divergence section the low-penetration chevron has negligible impact. When divergent angle is reduced (going from $\mathrm{M}_{\mathrm{d}}=1.65$ to $\mathrm{M}_{\mathrm{d}}=1.5$ ), the chevron has noticeable impact on flow.

The change in chevron impact is even more dramatic when viewed by axial vorticity, shown in Figure 15. On the G6 nozzle the low-penetration chevron registers no increase in the strength of the axial vortices over that shown in the baseline G6 nozzle (Figure 8). When applied to the G5 nozzle, the same chevron creates very strong vortices, very similar to the P05L13W08 chevrons on the G6 nozzle. Changing the divergence angle of the nozzle by $1.4^{\circ}$ causes the chevron to act as if it has over twice the penetration. 


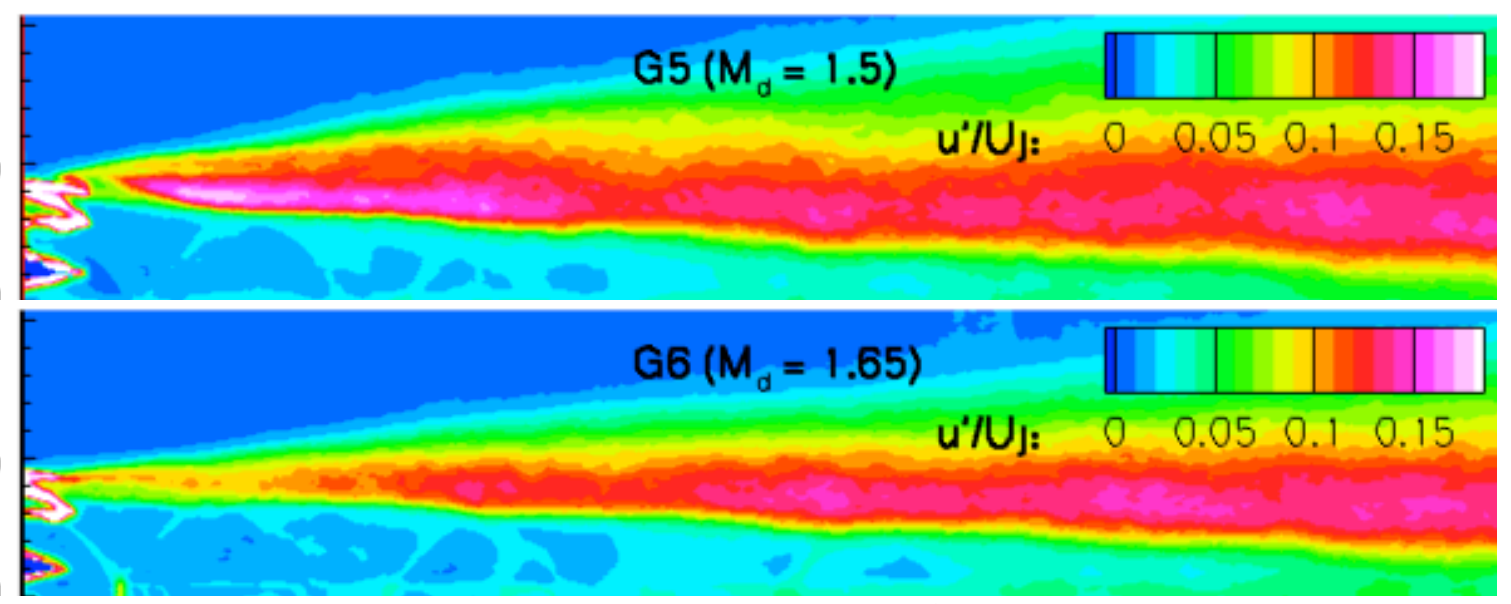

Figure 14 Streamwise cuts of streamwise turbulence intensity u'/Uj for chevron P02L13W08 mounted on nozzles with $M_{d}=1.5$ (nozzle G5) and $M_{d}=1.65$ (nozzle G6).

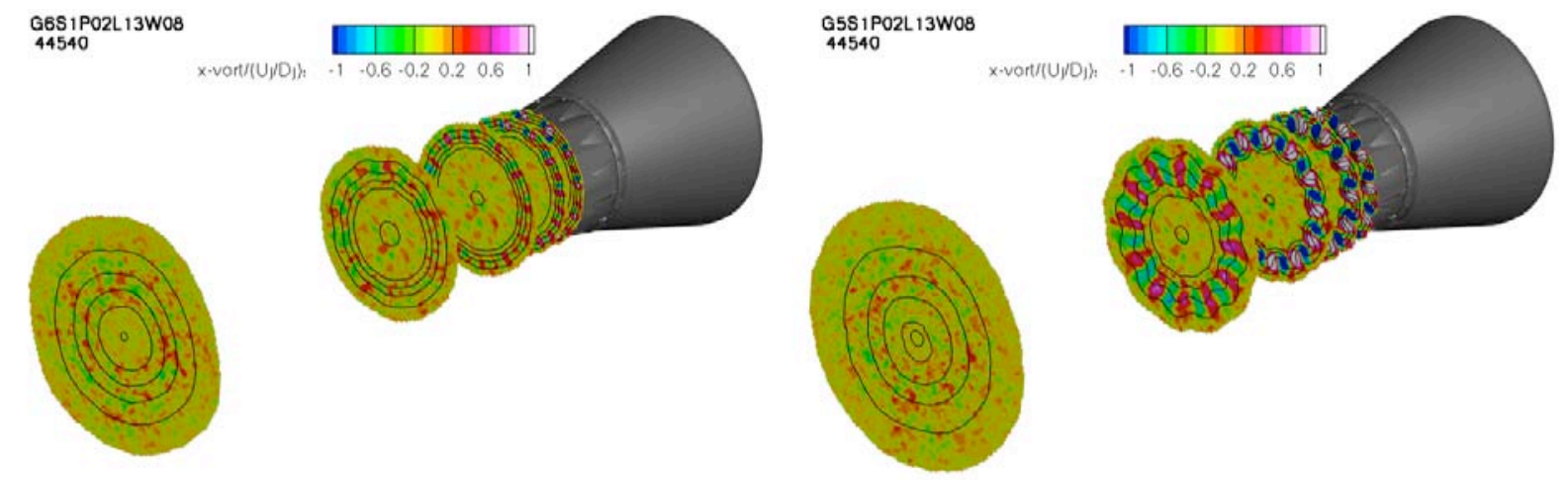

Figure 15 Cross-stream contours of mean streamwise velocity (black lines) and streamwise vorticity (color fills) for chevron P02L13W08 mounted on nozzles with $M_{d}=1.5$ (nozzle G5) and $M_{d}=1.65$ (nozzle G6). NPR $=3.2, T_{t, c} / T_{\infty}$ $=3.2, M_{\text {ideal }}=1.48$. Color fills cut off at $U / U_{j}=0.1$.

\section{Summary}

The turbulent velocity fields of scale-models of tactical aircraft nozzles have been measured using particle image velocimetry. The highly realistic model nozzles allowed variation in nozzle area ratio and incorporated a family of chevrons on their trailing edge. The chevron designs parametrically varied penetration, length, and width. Both streamwise and cross-stream planes of data were acquired for five chevron designs at realistic flow conditions of tactical aircraft, including the nozzle cooling flow. It was observed that the small bypass flow used as cooling flow in real nozzles produced a velocity deficit in the flow profile that encounters the chevrons; the impact on the enhanced mixing of the chevrons is debatable, however, because the increased density of the cold flow hitting the chevron may compensate for its reduced velocity. More generally, the impacts of chevron design on the jet plume have been documented and analyzed in terms of shock structure, centerline velocity decay and turbulent velocity. Penetration and length were key parameters for these chevron designs. As expected, more chevron penetration was required to have an effect on the overexpanded flows of tactical aircraft operating at low altitude than is required in subsonic convergent nozzles. It was also demonstrated that the effect of penetration varied with the varying of nozzle area ratio. The impact of chevrons on potential core length varied with Mach number relative to the nozzle design Mach number. Turbulence intensity in these supersonic jets was found to be comparable to that found in subsonic and single-stream jets. The impact of chevrons on turbulence was generally similar to that of subsonic jets-an increase in turbulence near the nozzle with a reduction in the higher, peak turbulence downstream around the end of the jet potential core. 


\section{Acknowledgements}

The authors would like to thank the team of technicians at NASA Glenn Research Center who so ably and professionally support the acquisition of the data contained in this report. The tests described in this report were supported by the Supersonics Project of NASA's Fundamental Aeronautics Program.

\section{References}

1 Seiner, J.M., Ukeiley, L. and Jansen, B., "Acoustic suppression associated with application of Chevrons to the trailing edge of a supersonic nozzle" Noise-Con Proc. Volume 112, Issue 1, pp. 262-271 (June 23, 2003).

${ }^{2}$ Henderson, B., and Bridges, J. (2010). "An MDOE Investigation of Chevrons for Supersonic Jet Noise Reduction," AIAA 2010-3926 (2010).

3 Frate, F.C. and Khavaran, A., "An Aerodynamic and Acoustic Assessment of Convergent-Divergent Nozzles with Chevrons," AIAA-2010-\#\#\#\#, 48th AIAA Aerospace Sciences Meeting, (2011).

4 Henderson, B. and Bridges J., "An MDOE Investigation of Chevrons for Supersonic Jet Noise Reduction,"AIAA-2010-3926, 16th AIAA/CEAS Aeroacoustics Conference, (2010).

5 Munday, D., Gutmark, E., Liu J. and Kailasanath, K. (2009) "Flow structure of Supersonic Jets from Conical C-D Nozzles," AIAA-2009-4005.

6 Khavaran, A. and Bridges, J. "Modelling of fine-scale turbulence mixing noise," J Sound Vibration, 279, 11311154 (2005). 